\title{
Características para una plataforma de información dirigida a profesionales que desean vivir y trabajar en el exterior basada en marketing experiencial y diseño de servicios
}

\section{Information platform characteristics based on experiential marketing and service design, aimed to professionals who wish to live abroad}

\section{Yady M. Guerrero Farfán (Colombia) \\ Maestría en Gerencia Estratégica \\ Institución Universitaria Politécnico Grancolombiano ymguerrero@poligran.edu.co}

\section{Resumen}

La necesidad de mejorar el nivel de vida por parte de colombianos calificados, motiva a buscar opciones fuera del país, pero el desconocimiento sobre procesos relacionados con ello es una brecha que inhibe que se de este fenómeno. Dado lo anterior, esta investigación tiene como propósito describir

\section{Sandra Patricia Rojas Berrío (Colombia)}

Doctora en Ciencias Administrativas Profesora de Tiempo Completo Universidad Nacional de Colombia sprojasb@unal.edu.co

\section{Abstract}

Competent Colombians need to improve their standard of living, motivates them to look for abroad options, but unawareness about related processes is a gap that inhibits this phenomenon. Therefore, this research aims to describe an information platform
Recibido:

Evaluado:

Aceptado:
26 de septiembre de 2016

12 de febrero de 2017

15 de abril de 2017

\section{PARA CITAR ESTE ARTÍCULO/TO CITE THIS ARTICLE}

Guerrero Farfán, Y. M. y Rojas Berrio, S. P. (2017)

Características para una plataforma de información dirigida a profesionales que desean vivir y trabajar en e exterior basada en marketing experiencial y diseño de servicios, Poliantea 13(24), pág. 63-84. 
las características de una plataforma de información para profesionales que quieren vivir y trabajar en el exterior desde la perspectiva del Marketing Experiencial y el Diseño de Servicios. Para tal fin, desde un enfoque social digital y de co-creación se realizaron entrevistas a profundidad y encuestas. Los principales hallazgos permiten afirmar que los usuarios quieren ser parte de la creación de este tipo de plataformas, conocer experiencias de otras personas y transmitir sus experiencias a otros. Así mismo, las características de la plataforma que mayor valoración tendrían entre los posibles usuarios son: ser actualizada, clara, rápida, confiable, veraz y amable.

Palabras Clave: Plataforma de información, Profesionales que desean vivir y trabajar en el exterior, Diseño de Servicios; Marketing Experiencial to it is a gap that inhibits this phenomenon. Because of the above this research aims to describe the characteristics of an information platform for professionals who want to live and work abroad from the perspective of Experiential Marketing and Design Services. To this end, from a digital social approach and co-creation depth interviews and surveys were conducted. The main findings are such that users want to be part of the creation of such platforms, meet other people's experiences and share their experiences with others. Likewise, the platform features that greater appreciation potential users would include being current, clear, fast, reliable, accurate and friendly.

Keywords: Information Platform; Live and Work Abroad as Professional; Service Design; Experiential Marketing 


\section{Introducción}

Autores como Guarnizo (2006), Ochoa (2012) y Retis (2013) dan cuenta de cómo importantes empresas quebraron entre los ochenta y noventa, debido a esta situación, muchos empleados fueron despedidos, incrementando así la tasa de desempleo; coincide lo anterior con que en estos años un promedio de 850.000 colombianos empezaron a buscar trabajo en otros países; surgiendo redes de apoyo de emigrantes. El primer país destino fue Estados Unidos, pero debido a restricciones legales, creadas por el gobierno norteamericano, pasaron a emigrar a lugares como Canadá, Europa, Centro América, al sur del continente y al Caribe, según cita Guarnizo (2006).

Retomando cifras del Banco Mundial en el 2011 y Colombia Nos Une en el 2010, en Estados Unidos, España, Venezuela y Ecuador viven el mayor número de emigrantes (Ramírez \& Mendoza, 2013). Así mismo, de la población de emigrantes en Estados Unidos más del 67\% está en edad para laborar, entre ellos más del $40 \%$ son personas mayores de
40 años y el $31 \%$ de los mayores de 25 años son profesionales. En países como España, los colombianos constituyen más del $8 \%$ de extranjeros totales en el país, mientras en la República Bolivariana de Venezuela hacen parte del $60 \%$ y en Ecuador representan el 62\% del total de emigrantes, según informa Ramírez \& Mendoza (2013).

Dado lo anterior se procederá a explicar los factores por los cuales los colombianos toman la decisión de viajar a trabajar y vivir al exterior. En primer lugar se encuentra la actual situación económica de Colombia, ya que no se puede desconocer que es un país en vía de desarrollo, en donde es mayor la demanda por parte de la fuerza laboral, que la oferta de vacantes. Se une a esta situación las pocas opciones de trabajo y los empleos no acordes al nivel profesional de los egresados, es por esto que los profesionales optan por trabajar en el exterior contribuyendo con la mejora de la economía al ingresar sus giros por remesas (Guarnizo, 2006).

El segundo factor consiste en la falta de orientación educativa del país, los 
jóvenes escogen su profesión sin tener en cuenta las necesidades de la nación, ni la disponibilidad de vacantes y el retorno de la inversión a realizar. Debido a esta descompensación entre lo que se requiere y lo que se necesita, los títulos no son valorados relata Didou (2008). Así mismo, en Coloma (2012) se observa que el $63 \%$ de colombianos que emigran con nivel de formación son profesionales y del 5\% han terminado una maestría y emigran a países como España, Brasil y México.

El tercer factor corresponde a la dificultad de ascenso y movilidad entre estratos socioeconómicos, escribe Hoyos (1981), asunto que a la fecha no se ha modificado dado que según Naciones Unidas el Índice de Desarrollo Humano (IDH), el cual es un indicador social estadístico compuesto por tres parámetros como vida larga y saludable, educación y nivel de vida digno; permite observar si una sociedad mejora las condiciones de vida de sus ciudadanos, es decir, a mayor cantidad de opciones mayor desarrollo humano; así mismo, para el 2013 en Colombia fue de 0,719 , ubicándola en el puesto 83 del IDH dentro de 187 países calificados, según PNUD (2013) y citado también por Ramírez y Mendoza (2013), lo que indica que no hay una mejor calidad de vida, comparado con otros países.

Así mismo, el cuarto factor es la política actual en que el sistema contrata en empleos no afines, se está educando para exportar trabajadores; por otro lado, otros países importan a los profesionales de acuerdo a las necesidades de crecimiento de su economía, según lo ex- presan trabajos como los de Chaparro \& Arias (1970).

En respuesta a la situación actual de búsqueda de empleo en otros países existen plataformas vía internet que proporcionan servicios, hasta ahora considerados necesarios para los usuarios, proporcionados por empresas nacionales, multinacionales, abogados expertos; las cuales asesoran a los profesionales para solicitar visa de trabajo o residencia en el exterior, sus oficinas se encuentran ubicadas en Colombia o en los países destino. Entre los procesos realizados vía web o por medio de oficinas están la evaluación del perfil, charlas instructivas, orientación durante el proceso de solicitud de visa de trabajo hasta la presentación de la entrevista en la embajada - no cubren a todos los países-.

Teniendo en cuenta las anteriores consideraciones y conociendo que se carece de portales o plataformas que contribuyan a cerrar la brecha de información de los profesionales calificados interesados en salir del país y mejorar su calidad de vida en el exterior, este trabajo se basa en la pregunta de investigación ¿Cuáles son las características para una plataforma de información dirigida a profesionales que desean vivir y trabajar en el exterior basada en Marketing Experiencial y diseños de servicios?

\section{Marco teórico}

Para que todo el proceso de vivir y trabajar en el exterior sea memorable se requiere planificar, organizar una infraestructura donde el profesional interactúe con la plataforma; por lo tanto, este 
apartado abordará los conceptos relacionados con Plataformas de Información, Marketing Experiencial y Diseño de Servicios.

Inicialmente, un plataforma de información es la forma automatizada de reunir, organizar y analizar datos, utilizando varios tipos de contenido, así el usuario obtiene un conocimiento preciso del mercado, para tomar decisiones de manera más rápida y eficiente sobre el mismo (Ainsbury, et al., 2000). Según, Wang, et al.(2010), la plataforma facilita la construcción de redes inteligentes donde se almacena información de forma segura; por lo tanto, es necesario conocer los usuarios para crear la arquitectura de la plataforma.

Ainsbury, et al. (2000) escriben sobre la recolección e integración de datos, dividiendo la plataforma en cuatro secciones principales: la primera es la recuperación de datos donde se crea un catálogo de búsqueda de información y recopilación de agentes que recuperan información, ajenos al usuario; la segunda, la clasificación y almacenamiento de datos obtenidos con anterioridad; la tercera, la información de navegación, consulta, análisis y creación de informes y herramientas de análisis; $y$ la cuarta, la integración de toda la información.

El Marketing Experiencial se enfoca en el estudio de las experiencias positivas o negativas que tiene el consumidor hacia personas o productos o servicios aporta Schmitt (2007). Aunado a lo anterior, según Verhoef, et al.(2009), para el cliente es más fácil recordar las marcas, servicios o productos si se encuentran relacionadas con sus emociones, sensaciones y vivencias (Gao, et al., 2012), concluyendo con Grönroos (2007) quien menciona la importancia de la positiva interacción de los clientes con productos y marcas, cada vez que se más favorable estos tendrán en su mente mayor significancia y las preferirán.

Reinoso Fernández-Caparrós (2011) relata que las emociones generan interés, atención y motivación, tanto hacia ciertos productos o servicios hasta crear sentimientos hacia ellos y hacer parte del estilo de vida del consumidor. Las empresas competitivas se centran en el cliente, en conocer sus experiencias, generan sentimientos, emociones, según Hartono, et al.(2012). Además, Sirapracha \& Tocquer (2012, citado en Manosso, et al., 2013 ) investigan sobre la lealtad y la experiencia recibida, encontrando que los clientes son más leales a medida que reciben experiencias positivas.

Estudios realizados sobre las experiencias de servicio como el de Maklan (2012) permite medirlas por medio de técnicas exploratorias, por otro lado Beaudoin (2014) analiza las experiencias del cliente estudiando sus conceptos, inspiraciones, recuerdos, el pensamiento crítico, las emociones, el compromiso, la comercialización, la conexión social, la traducción y la confianza.

El Diseño de Servicios permite observar que con el paso de los años los clientes son más heterogéneos, sus necesidades son diferentes, así este debe combinar óptimamente la demanda de usuarios 
con su oferta, comentan Baltas, et al. (2013), por lo tanto se requiere diversificar las ofertas de servicios, resaltando sus atributos y teniendo un abanico de posibilidades para los diferentes clientes.

Ariely, et al. (2013) relatan que en la actualidad el internet facilita personalizar los servicios de acuerdo a las necesidades del cliente, además permite conocerlo, aprender de él y generar lealtad hacia la marca. El internet integra a los clientes, competencia, proveedores de afuera hacia adentro, afirman autores como Kalakota \& Robinson (2003), además que posibilita utilizar información para combinarla y publicarla escriben Diaz, et al. (2012), así, esta información permite crear bases de datos como el CRM, el cual ayuda a conocer mejor sobre los gustos y necesidades de los clientes y mejorar los procesos internos de la empresa (Rossiter, 2012).

El marketing experiencial y el diseño de servicios tienen puntos en común para tener en cuenta en el presente estudio, con el fin de conocer las tendencias actuales, y así determinar las características de la plataforma de información, estos se explican a continuación:

\section{Redes sociales}

El marketing experiencial para generar experiencias memorables, según Gao, et al. (2012), se apoya en redes sociales, con el fin de que el usuario tenga una identidad social. El estilo de vida lo generan actividades, intereses, gustos y opiniones alrededor de prototipos. El cliente se siente satisfecho no solo por el producto o servicio recibido, sino por el significado que tienen dentro de su entorno, escribe Kotri (2011). Por otro lado, el diseño de servicios investiga las consultas que realizan las personas vía web, los cuales permiten generar información, según Buur, et al. (2013), y conociéndo los gustos que son públicos a través de la red se facilita el diseños de un servicio, escriben Chiu \& Tomimatsu (2013) y Yu, et al. (2012).

Las estrategias de marketing aprovechan los medios de comunicación que unifican prototipos sociales, dicen Lee C., et al. (2014), y utilizando tácticas como la segmentación de mercados, se llega a conocer los usuarios de las redes sociales. Este conocimiento permite comprender las demandas variables y así mejorar la calidad del servicio, explica Jamali, et al. (2013). La tecnología móvil, dice Walravens (2012), permite una interacción amigable, facilidad de uso, novedad en diseños y generan el boca a boca, la información obtenida de ellas y la innovación en la tecnología facilitan la innovación empresarial, comenta Mager, et al., (2014).

\section{Co-creación}

En Marketing Experiencial existen autores como Schmitt (2007) quien escribe sobre la importancia de que los usuarios opinen acerca de sus productos y participen en la co-creación al mejorar los actuales o proponer nuevos, de esta forma estos serán acordes a las expectativas del cliente. La experiencia de cada cliente es mejor al participar en la co-creación de sus productos o servicios, comenta Garg, et al., (2012). Según Grônroos (2007), para que una empresa 
tenga ventaja competitiva, la oferta de servicio debe ser completa y quien más que el cliente para conocer como debe ser dicha oferta.

Las empresas líderes o competitivas deben evolucionar a la par de las necesidades del cliente, para esto los productos o servicios deben ser más personalizados, esto lo estudian Kalakota \& Robinson (2003) y Lu \& Bao (2012). Por otro lado, Yeh H., et al. (2013) y Eichentopf (2011), analizan que un buen servicio fija la atención en el cliente como agente innovador, participante co-creador en todo el proceso. Autores como Lefebvre (2012) relatan que los servicios deben sufrir transformación e innovación por parte del usuario. Chen, et al. (2013), escriben sobre la innovación de servicios por medio de tecnologías de la información generadoras de valor.

Como se puede observar el Marketing Experiencial y el Diseño de Servicios son conceptos con puntos afines, por lo tanto algunos autores han realizado investigaciones basados en estos dos, tales como:

Dixon, et al. (2014) resaltan la importancia de la coproducción del cliente, aplicado al diseño de servicios y experiencias en los cuales el cliente sea partícipe. A su vez, para el caso de las prácticas realizadas para teléfonos inteligentes en Taiwan se analiza la relación por medio de un diseño de un servicio de hardware y software, entre el valor percibido por el cliente, la calidad y el valor social del precio (Hsiao, 2013), en suma, se evidencia la posibilidad de crear servicios acordes a la percepción del cliente y a estos se les asocia un precio de acuerdo a la calidad que considera el cliente posee.

Los clientes finales deben participar activamente tomando la posición de coproductores, para examinar los elementos que facilitan la interacción interpersonal en el servicio. La sociabilización facilita la disciplina de diseño en grupos (Cho, 2011), a un estudio efectuado en el ámbito del m-Commerce permite el diseño de herramientas para transacciones en dispositivos móviles por medio de la sociabilización de experiencias de usuarios (Yan \& Qiong, 2011).

Pasman (2011) valida el cómo en la comunicación crossmedia la información se comparte en múltiples plataformas en donde el usuario tiene vivencias asociadas a conocer globalmente información de varios contenidos, de esta forma se diseñan aplicaciones según las necesidades y hábitos del consumidor en los sitios visitados. Así mismo, cinco experimentos de simulación de experiencias de servicio en los dominios de viajes en línea y las citas en línea realizados por Buell \& Norton (2011) comprueban la importancia del valor de servicio percibido, donde se inducen sentimientos de reciprocidad con transparencia en la operación al ofrecer varias alternativas al cliente.

Otros autores como Wu (2011) explican que la satisfacción no se traduce en lealtad, hay que tener en cuenta la comodidad, las relaciones interpersonales; todo esto se obtiene por la experiencia y la credibilidad de los servicios que se ofrecen. La búsqueda de 
atributos por experimentar la credibilidad, la comodidad y el compromiso para retener clientes, generan relaciones estrechas con estos. Cuando se reciben servicios de alta calidad el usuario desea experimentar dicha calidad con todos sus proveedores (Riascos \& Gonzalez, 2008).

El Kansei Engineering (KE) es una metodología que permite la interpretación y traducción de las emociones de los clientes en parámetros de diseño, abarca tanto los productos y los servicios, tiene en cuenta los atractivos exteriores y optimiza las propiedades que no son fácilmente detectables o visibles, este modelo se aplicó en hoteles de lujo para turistas indonesios, japoneses y de Singapur (Hartono, et al., 2012).

Se observa que existen aplicaciones de Marketing Experiencial y el Diseño de Servicios, pero aún no existen para una plataformas de información dirigida a profesionales que quieran vivir y trabajar en el exterior, por lo tanto es necesario antes de crearlas conocer las características principales que deben poseer, lo que visualiza el vacío que justifica esta investigación, la cual tiene como objetivo establecer las características para una plataforma de información dirigida a profesionales que desean vivir $y$ trabajar en el exterior basada en Marketing Experiencial y Diseño de Servicios.

\section{Metodología}

Para cumplir el objetivo general se plantearon los siguientes objetivos específicos: revisar la literatura concerniente a los factores por los cuales los profesionales emigran al exterior, a Plataformas de Información, Diseño de Servicios y al Marketing Experiencial, que se presentó en el apartado anterior; así mismo, explorar las características que debería tener una plataforma de información dirigida a profesionales que quisieran por primera vez vivir y trabajar en el exterior; para finalmente, determinar las características relevantes para una plataforma de información dirigida a profesionales que quisieran por primera vez vivir y trabajar en el exterior.

El enfoque metodológico se aborda desde la cosmovisión pragmática a partir de un diseño descriptivo mixto transversal. A su vez, la investigación fue de carácter secuencial exploratoria (Creswell, 2003) con respecto a los momentos en que se aplicaron los instrumentos toda vez que la metodología contó con las siguientes dos fases en respectivo orden:

\section{Fase cualitativa}

Se tuvo en cuenta la literatura y fuentes secundarias como las plataformas de información para personas que desean vivir en el exterior ubicadas en la web para generar las preguntas base, aunque se desconocían las características de una plataforma de información para profesionales. Para esto fue necesaria la recolección de información por medio de entrevistas personales a profundidad. Se entrevistaron a los profesionales de la ciudad de Bogotá por facilidad de ubicación y por poseer el mayor número de emigrantes, según estudios realizados por el DANE (2014); además, 
porque la capital tiene el mayor porcentaje de graduados del país, cerca de un 38\% (Observatorio Laboral para la Educación, 2014).

Se estudiaron profesionales (hombres o mujeres) en edades entre 25 y 45 , tomando como referencia de país a Estados Unidos donde se encuentra la mayoría de población emigrante, aproximadamente un $34.6 \%$. De los cuales los profesionales corresponden a un $67 \%$, entre ellos más del $71 \%$ son personas entre 25 a 40 años de edad (Ramírez y Mendoza, 2013). Los profesionales fueron entrevistaros en dos grupos, el primero conformado por 5 personas que ya habían viajado y trabajado en el exterior y luego 5 personas que deseaban tener por primera vez dicha experiencia, cantidades con las que se logró saturación de categorías, es decir, no se encontraban categorías nuevas ya que los datos se convierten en repetitivos o redundantes (Kornblit, 2007).

Para realizar el análisis hermenéutico de las entrevistas, estas fueron registradas por escrito y audio, se reprodujeron con fines de transcripción, posteriormente se codificaron teniendo en cuenta las variables - Profesionales en el Exterior, Marketing Experiencial, Diseño de Servicios, Plataforma de Información-y sus respectivas categorías, para así proceder a realizar representaciones de familias y códigos e identificar las comunalidades - involucramientos en grupos de códigos y categorías- en Atlas.ti. 7.0, el cual es un software para análisis y procesamiento de datos cualitativos, este permite agrupar y graficar los resultados de las entrevistas de acuerdo a la fundamentación hermenéutica y afinidad, para así unificar los conceptos (Huberman \& Miles, 1994).

\section{Fase cuantitativa}

Partiendo de la información suministrada por las entrevistas, se agruparon las preguntas de acuerdo a los temas de interés en categorías reagrupadas lógicamente, las cuales están enfocados a los objetivos del presente estudio; el primer grupo correspondia a Marketing Experiencial; el segundo, las características de las plataformas de información en general; el tercero, factores por los cuales se emigra a otros países y el cuarto el diseño de servicio de la plataforma para profesionales que desean vivir y trabajar en el exterior. Se estructuraron la preguntas en escala de likert, de tal forma la primera parte constó de 3 preguntas, la segunda 3 , la tercera 1 y la cuarta de 11 .

Cabe destacar que, para las encuestas se tuvo en cuenta la misma población y filtros. El tamaño de la población de profesionales en Colombia desde 1986 al 2010 - personas que actualmente tienen 25 a 45 años- es aproximadamente de 3.200.000, informa el Ministerio de Educación NacionalRepública de Colombia (2012), los rangos de edades de los profesionales son los mismos que explicamos en la fase cualitativa (Ramírez \& Mendoza, 2013). La muestra se seleccionó circunstancialmente teniendo en cuenta lugares de mayor afluencia de profesionales provenientes de todo el país en Bogotá.

No obstante, el tamaño muestral se calculó con la fórmula para calcular mues- 
tras en poblaciones infinitas (Mateu \& Casal, 2003) con un 5\% de margen de error y un $99 \%$ de nivel de confianza y probabilidad de éxito y fracaso del 50\%, lo que dio un valor de muestra de 396 profesionales, para mayor confiabilidad de este estudio y teniendo en cuenta que pudieren algunos datos tener que eliminarse de la base de datos fueron efectuadas 422 encuestas, las cuales se realizaron durante 28 días con un promedio de 15 por día. Para el análisis de resultados, después de haber tabulado en Excel la información cuantitativa se usó el software IBM Statistics, se tomaron según este software como variables nominales el género, variables de escala como la edad y variables ordinales en en escala Likert.

Se aplicaron encuestas piloto a 30 profesionales, con el fin de confirmar la consistencia interna del instrumento, de esta forma la prueba de Alfa de Cronbach arrojó una confiabilidad del 0.979 , para un promedio de edades de 35 años con desviación estándar de 6.102; lo que significa que hay una consistencia interna, con el resultado obtenido es fiable el instrumento al estar más cercano al uno. Posteriormente se aplicaron las 422 encuestas con un resultado definitivo de Alfa de Cronbach de 0.979, corroborando la consistencia interna inicial que tenia la encuesta piloto.

\section{Resultados}

La organización de este apartado responde a los objetivos específicos que se plantearon para cumplir con el propósito principal de la investigación, excepto el de revisar la literatura con- cerniente a los factores por los cuales los profesionales emigran al exterior, a Plataformas de Información, Diseño de Servicios y al Marketing Experiencial, dado que éste se presentó en el apartado previo de revisión de literatura de este documento. Es así que, a continuación se presentan los resultados de los demás objetivos.

Exploración de las características que debería tener una plataforma de información dirigidar a profesionales que quisieran por primera vez vivir y trabajar en el exterior

Este objetivo de investigación se llevó a cabo a través de la realización de 10 entrevistas a profundidad, tal como se indicó en el método de este documento, la información se procesó en Atlas.ti. En este orden de ideas, el análisis hermenéutico permitió corroborar que las variables revisadas en la literatura se encuentran relacionadas entre sí a partir de los ejes de indagación que se representan como códigos axiales de las familias identificadas.

En primera instancia, se encuentra que el Diseño de Servicio para la plataforma de información de profesionales incluye aspectos como: soporte en la viabilidad de la migración con evaluación y retroalimentación previa, soporte en el manejo de idiomas, asesoría y orientación previo y durante la migración, soporte en empleabilidad y creación de empresas en el exterior; así como que el diseño debe incluir aporte de y generación de experiencias enriquecedoras de otras culturas y la creación de escenarios de co-creación. Estas dos 
últimas variables se encuentran relacionadas con los factores para emigrar del país y el marketing experiencial, la figura 1 presenta estas relaciones.

Así mismo, al profundizar en el eje de indagación referido a las características puntuales que debe tener la plataforma para el ámbito de los profesionales que quisieran por primera vez vivir $\mathrm{y}$ trabajar en el exterior, se encuentra que e- xisten características relevantes para los participantes en la plataforma: confiabilidad y seguridad de la información, rapidez, gratuidad y amigabilidad. No obstante, las más relevantes en orden hermenéutico son: información pertinente, soporte en la viabilidad de la migración con evaluación y retroalimentación previa y generación de escenarios de Co-creación, la figura 2 representa esta información.

Figura 1. Relaciones existentes entre el Diseño de Servicio, el Marketing Experiencial y los factores para emigrar en el exterior

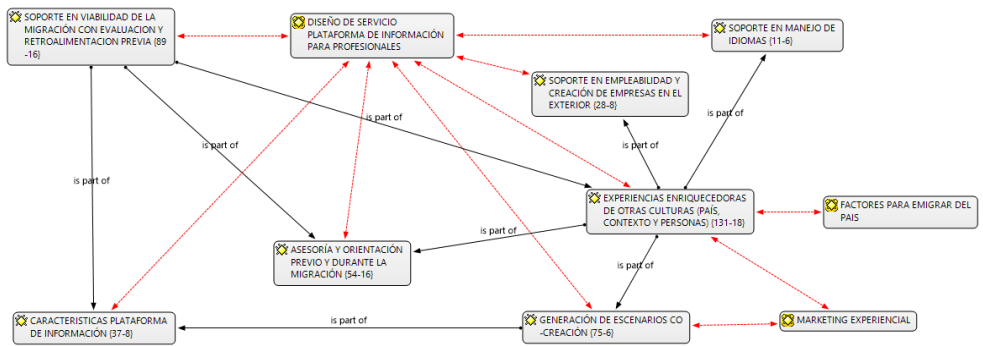

Fuente: elaboración propia a partir del procesamiento de las entrevistas en Atlas.ti

Figura 2. Características de la Plataforma de Información

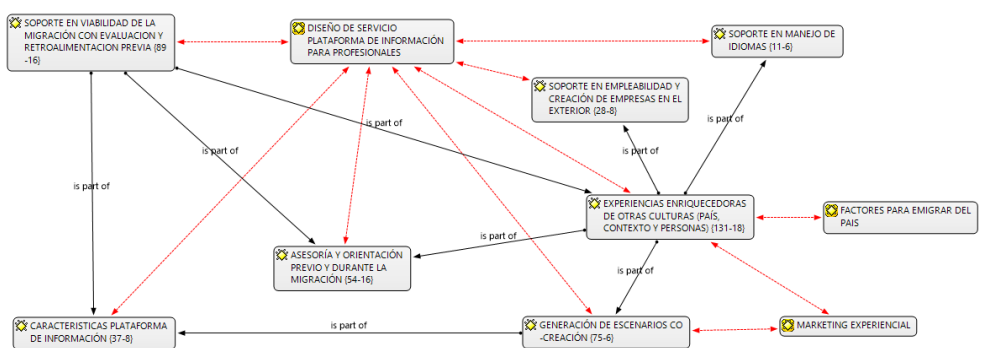

Fuente: elaboración propia a partir del procesamiento de las entrevistas en Atlas.ti

En este orden de ideas, en cuanto a la información pertinente los profesionales buscan que la plataforma brinde información del país en cuanto al orden social, cultural, legal y económico, a continuación se presentan discursos de los participantes que ilustran esta necesidad:
"Sería ideal tener ...Información sobre hospedajes, vivienda ....características y costumbres del país, alimentación, como movilizarse en la ciudad ...socializar con otras personas"

“...cómo apostillar ....información sobre turismo, guía o traductor 
...conocimiento costumbres habitantes locales"

Así mismo, los participantes esperan que la plataforma brinde soporte en la viabilidad de la migración con evaluación y retroalimentación previa. Lo anterior, implica desde pruebas psicotécnicas pasando por ensayos en entrevistas laborales, entre otros. De igual forma, respecto a la generación de escenarios de Co-creación es necesario que la plataforma brinde espacios para retroalimentarse y re-inventarse a partir de las opiniones y necesidades de los usuarios. Es importante destacar que este aspecto también está relacionado con la variable Marketing Experiencial.

Identificar las características relevantes para una plataforma de información dirigida a profesionales que quisieran por primera vez vivir y trabajar en el exterior

Este objetivo de investigación se llevó a cabo en dos partes, la primera de orden univariado y la segunda multivariado. En este orden de ideas, el análisis univariado indica que la distribución de frecuencias de los encuestados tuvieron edades por debajo de 41 años, existen edades que tuvieron mayor cantidad de encuestados como 28 años con 42 encuestas y la de 30 años con 35, seguido la edad de 29 con 32 . Así mismo, el 56\% de los encuestados eran mujeres mientras que el $43 \%$ eran hombres.

Los encuestados en más de un 50\% estuvieron de acuerdo en todas las preguntas a excepción de las relacionadas con participar en grupos de actividades cultur- ales, religiosas; también no estaban de acuerdo con ingresar a redes sociales por curiosidad, publicar fotos y tener contactos mundiales; además, que el ingreso a plataformas fuera por moda, por confianza, por deportes, así como, el tener dentro de las plataformas chats, estrellas para calificar, publicidad, conocer sobre transacciones.

Se consideraría como las características más representativas las que superaran la media, es decir, dentro de la calificación en la escala de Likert de 1- 5, fueron calificados por encima de 4.02 , ya que estas respuestas tendrían un promedio mayor de acuerdo o totalmente de acuerdo, estás se tienen en cuenta para el análisis bivariado toda vez que el objetivo se concentra en evidenciar las que tienen relevancia.

Así mismo, la mediana de las variables estuvo por encima de 4 a excepción de las preguntas a cerca de los grupos religiosos y compañeros de trabajo, publicar fotos en redes sociales, tener contactos mundiales, ingresar a plataformas por moda o por generar confianza, que la plataforma tuviera estrellas para calificar, publicidad; en estas mismas respuestas la moda fue menor o igual a 3. Los datos variaron con respecto de la media similarmente a excepción de las edades donde la desviación estándar fue de 5.6.

Entonces, estuvieron por encima de la media preguntas como el prototipo social más aceptado es la familia, además la importancia de plataformas de información que sean organizadas, actualizadas, claras, confiables, rápidas, ve- 
races, tengan fácil de uso, buena presentación, noticias y servicios gratuitos, sean fáciles en navegación y en accesibilidad; además, posean información fácil de buscar, segura y fácil de enviar. Entre los factores por los que viajan las personas al exterior están mejorar el nivel de vida y profesional. Por otro lado, se requiere soporte en la viabilidad de la migración con evaluación para que conozcan si aplican a la visa se requiere que la plataforma sea actualizada, rápida, clara, que explique los objetivos de la misma, sea confiable, se realice directo de la página y aplique toda la familia.

La orientación de la visa vía web necesita ser completa, clara, exacta, sencilla, verdadera y confiable; debe tener los requisitos de documentación, económicos y de tiempo, entidades donde dirigirse, información de las diferentes clases de visas; además requiere un asesor virtual. La asesoría para la entrevista en la embajada se requiere sea personal, se conozca conocer los objetivos claros, información del proceso exacto, conocer los resultados correctos e incorrectos, retroalimentación y acompañamiento postentrevista. Los descuentos más llamativos fueron en tiquetes, hoteles, alimentación, seguros, educación, transporte y vivienda.

La recepción en la llegada al país debe ser confiable, amable, sencilla, debe tener intérprete, recibirse desde el aeropuerto y transportarlo hasta el lugar destino. La asesoría dentro del país destino se requiere antes de llegar en los temas de licencia de conducción, salud, educación, proceso de cambio de visa, homologación de carreras, derecho-civil y laboral. El servicio de inmobiliaria necesita ser rápido, cómodo, ofrecer varias opciones, con fotos, contactos, calificar sitios, dar mapas de información, informar del tráfico, segmentado por cánones, con asesoría de formas de pago y asesoría en compra de bienes.

El traslado dentro del país requiere ser personalizado, además de tener opciones según el presupuesto, con guía turístico, gps, mapas de sitios de interés y rutas de transporte público. Los cursos de idiomas en la página deberían tener un componente de práctica vía teleconferencia, personalizado; para el país destino requiere tener el listado de cursos avalados, ubicación por localidad, calificación de lugares, requisitos, presupuestos, contacto, horarios y transporte.

La bolsa de empleo debe calificar el perfil, realizar entrevista virtual, dar información sobre experiencia, salarios, requisitos por sector y por ubicación geográfica; además de información por afinidades. La orientación para crear empresas debe ser según las necesidades de productos o servicios, conocimiento de normas del país, seguridad del dinero, facilidad para reunir emprendedores, informar de empresas ya exploradas, investigación de mercado del país, información de leyes y regulaciones, fuentes de financiación, instituciones de apoyo e información por sectores económicos.

Sin embargo, teniendo en cuenta que las anteriores medidas no son concluyentes o más bien podrían considerarse como arbitrarias para determinar la relevancia 
de las características, se ejecutó un análisis factorial exploratorio con extracción de máxima verosimilitud y rotación varimax.

Lo anterior, con el fin de reducir al máximo las variables que mejor explican la varianza, lo que en otras palabras es maximizar ésta última. Es así que, lo resultados permiten determinar las características de la plataforma que son más relevantes para la población bajo estudio, toda vez que a partir de la prueba KMO y el test de esfericidad de Barlett permiten afirmar que es viable realizar el análisis factorial, y a su vez que la medida de adecuación de los datos es excelente por estar por encima de 0,9 , con un resultado de 0,935 , la tabla 1 muestra los resultados.

Tabla 1. Prueba KMO y el test de esfericidad de Barlett

\begin{tabular}{|l|l|r|}
\hline \multicolumn{2}{|l|}{$\begin{array}{l}\text { Medida Kaiser-Meyer-Olkin de } \\
\text { adecuación de muestreo }\end{array}$} & 0.935 \\
\hline $\begin{array}{l}\text { Prueba de esferi- } \\
\text { cidad de Barlett }\end{array}$ & $\begin{array}{l}\text { Aprox. } \\
\text { Chi-cuadrado }\end{array}$ & 59348,095 \\
\cline { 2 - 3 } & Gl & 16290 \\
\cline { 2 - 3 } & Sig. & 0.000 \\
\hline
\end{tabular}

Fuente: elaboración propia a partir del procesamiento de las encuestas en IBM Statistics

En este orden de ideas, los resultados permiten dar cuenta que el $72 \%$ de la varianza total explicada ésta en 40 factores; no obstante, en el primero de ellos se encuentra en $29 \%$ de la varianza y el segundo sólo aporta $4.9 \%$ más. Es así que, para efectos de determinar las características relevantes para una plataforma de información dirigida a profesionales que quisieran por primera vez vivir y trabajar en el exterior se tomarán aquellas variables que en el primer factor cuentan con cargas superiores a 0.65 . La tabla 2 presenta los resultados en orden descendente.

Tabla 2. Características relevantes para una plataforma de información dirigida a profesionales que quisieran por primera vez vivir y trabajar en el exterior

\begin{tabular}{|c|c|c|c|c|c|}
\hline Característica & Carga Factorial & Característica & Carga Factorial & Característica & Carga Factorial \\
\hline \hline Orientacion/R10 & 0,766 & Característica/R3 & 0,697 & Característica/R27 & 0,664 \\
\hline Orientación/R2 & 0,754 & Característica/R2 & 0,693 & Orientación/R7 & 0,663 \\
\hline Orientación/R11 & 0,749 & Encuesta Filtro/R3 & 0,691 & Idiomas/R2.3 & 0,662 \\
\hline Orientación/R4 & 0,748 & Empresas/R1 & 0,690 & Ases País/R5 & 0,659 \\
\hline Recepción/R2 & 0,742 & Serv Inmo/R1 & 0,688 & Empresas/R6 & 0,659 \\
\hline Orientación/R8 & 0,730 & Característica/R17 & 0,687 & Empresas/R3 & 0,659 \\
\hline Orientación/R1 & 0,729 & Serv Inmo/R13 & 0,684 & Idiomas/R2.5 & 0,657 \\
\hline Orientación/R5 & 0,717 & Recepción/R5 & 0,674 & Ases Entre/R4 & 0,657 \\
\hline Serv Inmo/R2 & 0,708 & Recepción/R1 & 0,673 & Empresas/R2 & 0,656 \\
\hline Orientación/R1.3 & 0,708 & Idiomas/R2.1 & 0,671 & Recepción/R3 & 0,656 \\
\hline Encuesta Filtro/R2 & 0,704 & Encuesta Filtro/R9 & 0,670 & Empresas/R9 & 0,655 \\
\hline Característica/R5 & 0,699 & Característica/R18 & 0,667 & Característica/R6 & 0,652 \\
\hline Encuesta Filtro/R8 & 0,699 & Serv Inmo/R3 & 0,666 & Empleo/R4 & 0,651 \\
\hline
\end{tabular}

Fuente: elaboración propia a partir del procesamiento de las encuestas en IBM Statistics

Entre las características más relevantes de una plataforma de información se encuentran el que sea actualizada, clara, rápida; facilidad en uso, en navegación en acceso y en envío. Por otro lado, la evaluación de la viabilidad para saber si las personas aplican a la visa se requiere que sea rápida, clara y confiable. La orientación para obtener la visa vía web necesita ser completa, clara, 
exacta, sencilla, verdadera y confiable; además, necesita los requisitos de documentación y de tiempo e informar sobre las entidades a donde dirigirse. A su vez, la asesoría para la entrevista en la embajada debe comprender como proceso el reconocimiento de los objetivos del viaje por parte del usuario del servicio. Para el servicio de recepción en la llegada al país, se obtuvo que éste debe ser confiable, amable, sencillo y algunos participantes indicaron la necesidad de contar con un intérprete.

Según los participantes, la asesoría cuando ya se esté dentro del país destino debería incluir asuntos para acceso a educación y procesos de formación adicionales a aquellos con los que cuente el usuario. Por otro lado, el servicio de inmobiliaria debe ser rápido, cómodo, ofrecer varias opciones, con asesoría de formas de pago. Los cursos de idiomas en la página necesitan incluir práctica en el país destino, tener el listado de cursos avalados, calificación de lugares y presupuestos. En el servicio de información de la bolsa de empleo requiere contener información precisa acerca de salarios e incentivos o condiciones laborales. La orientación para crear empresas debe ser según las necesidades de productos o servicios, conocimiento de normas del país, seguridad del dinero, investigación de mercado del país e instituciones de apoyo.

A fin de hacer un contraste entre los resultados de orden cualitativo y los cuantitativos se encuentra que, en primera instancia, existen similitudes en la variable (o categoría orientadora) Diseño de Servicio para la Plataforma de Infor- mación de Profesionales. En este contexto, se requieren aspectos como: Soporte en la Viabilidad de la migración con evaluación y retroalimentación previa, soporte en el manejo de idiomas, asesoría y orientación previo y durante la migración, soporte en empleabilidad y creación de empresas en el exterior. Así mismo, existen aspectos a tener en cuenta al conocer como requieren dichos servicios los usuarios como lo son confiabilidad y seguridad de la información - en el dinero-, rapidez; además es importante que toda la información sea pertinente.

No obstante, en segunda instancia tanto la perspectiva cualitativa como la cuantitativa difieren en que para la variable de Diseño en la fase exploratorio se encontró que para la plataforma era necesario: la generación de experiencias enriquecedoras de otras culturas, el interés por tener servicios gratuitos y la amigabilidad, asuntos que no resultan relevantes en la validación empírica de la encuesta.

\section{Conclusiones}

Esta investigación permitió describir las características de una plataforma de información para profesionales que quieren vivir y trabajar en el exterior desde la perspectiva del Marketing Experiencial y el Diseño de Servicios, las cuales se constituyen en: la encuesta filtro, orientación visa vía web, asesoría entrevista, descuentos, recepción en el país destino, asesoría dentro del lugar destino, servicios de inmobiliaria, traslado en el lugar destino, curso de idiomas, bolsa de empleo y orientación para la creación de empresas. 
Es relevante destacar que el desarrollo de las entrevistas a profundidad y el subsecuente análisis hermenéutico se realizó con el fin de verificar las variables de la literatura y diseñar los ítems del cuestionario. Se encontraron categorías emergentes con respecto a la literatura previa como el hecho que dentro de las redes sociales existen lazos fuertes como los familiares y estos son influenciadores en el momento de consultar información. Así mismo, dentro de las plataformas de información las más utilizadas son las de redes, mismas que son resultado de la co-creación por parte de los usuarios. Por otro lado, se comprueba que el diseño de un servicio implica tener en actualización continua, claridad, rapidez, confiabilidad, veracidad y amabilidad.

Con respecto a los aportes de Guarnizo (2006), Ochoa (2012), Retis (2013) y Ramírez y Mendoza (2013) en cuanto a los factores por los cuales los profesionales emigran al exterior, se confirma la necesidad por parte de los profesionales del país de una plataforma de información para vivir y trabajar en el exterior.

Adicionalmente, en lo que respecta al Marketing Experiencial, de acuerdo a los resultados obtenidos, los participantes buscan experiencias enriquecedores de otras culturas (país, contexto y personas), para esto requiere soporte en manejos de idiomas, en temas de empleo y creación de empresas en el exterior y antes de hacer parte de dicha experiencia demandan saber previamente si son aptos o no, para posteriormente ser orientados en todo el proceso y hacer parte de la experiencia co-creando. En cuanto al Diseño de Servicios se concluye que el usuario requiere soporte antes de iniciar el proceso para conocer si se es apto o no, además de retroalimentación, asesoría durante todo el servicio y hacer parte de la generación de escenarios (cocreación).

Desde el punto de vista de co-creación toda la información inicialmente suministrada en la entrevista permitió corrobar lo indicado por Schmitt (2007), en cuanto a que se deben crear servicios de forma colaborativa, con el fin de cumplir con todas sus expectativas. Si existen ya varias plataformas, una forma de ser competitivos es conocer lo que el cliente requiere y crear servicios según sus necesidades y acordes a las experiencias que desean obtener. Incialmente, se tendrían en cuenta las características conocidas en este trabajo, teniendo presente que la humanidad evoluciona y las necesidades varian.

Cabe destacar que el presente trabajo sirve de partida para futuras investigaciones como el análisis y validación de las dimensiones dentro de las interacciones en plataformas para éste ámbito, además de investigar que tipo de aplicaciones se podrían crear, o cómo podría ser la arquitectura de dicha plataformas y en qué participarían o co-crearían los usuarios.

Finalmente, este trabajo tuvo limitaciones externas como: la carencia de actualización de los registros de los profesionales graduados de las universidades que pudo haber sido un marco muestral más preciso y el registro de profesionales que desean vivir y trabajar en 
el exterior, al momento del estudio; así como la falta de información sobre la cantidad de profesionales que emigran por país de destino. Así mismo, una limitación interna de esta investigación es que el diseño para la fase cuantitativa fue de carácter transversal, lo que se convierte en una oportunidad para que futuras investigaciones aborden este problema longitudinalmente.

\section{Referencias}

Ainsbury, R. D. y otros, (2000). Method And Apparatus For Performing Data Collection, Interpretation And Analysis, In An Information Platform. United States, Patente n ${ }^{\circ} 6,078,924$.

Ariely, D., Bitran, G., Rocha, P. \& Oliveira, (2013). Design to learn: customizing services when the future matters. Pesquisa Operacional, pp. 33(1), 37-61.

Baltas, G., Tsafarakis, S., Saridakis, C. \& Matsatsini, (2013). Biologically Inspired Approaches to Strategic Service Design: Optimal Service Diversification Through Evolutionary and Swarm Intelligence Model. Journal of Service Research, pp. 2, 16, 186, 201.

Banco de la República, (2012). Evoluciòn de la Balanza de pagos enero a junio de 2012, Bogotá: s.n.

Beaudoin, J., (2014). A framework of image use among archaeologists, architects, art historians and artists. Journal of Documentation, pp. 1, 70, 119, 147.
Buell, R. \& Norton, M., (2011). The labor illusion: How operational transparency increases perceived value. Management Science, $p$. $9,57,1564,1579$.

Buur, J., Ankenbrand, B. \& Mitchell, R., (2013). Participatory business modelling. CoDesign, pp. 1, 9, 55,71 .

Chaparro Osorio, F. \& Arias Osorio, E., (1970). La emigración de profesionales y Técnicos Colombianos.

Chen, C.-L., Lin, R. \& Zhang, A.F., (2013). Constructing a Service Innovation Model for Creative Industrial Parks. International Journal of Arts and Commerce, pp. 151-165.

Chiu S., -C. \& Tomimatsu, K., (2013). User involvement in idea brainstorming of design process: Finding the effective strategy in social network service. Lecture Notes in Computer Science (including subseries Lecture Notes in Artificial Intelligence and Lecture Notes in Bioinformatics), pp. 593, 598.

Cho E, -J., (2011). Interpersonal interaction for pleasurable service experience. DPPI'11 - Designing Pleasurable Products and Interfaces, Proceedings.

Creswell, J. (2003). Research Design: Qualitative, Quantitative, and Mixed Methods Approaches. Thousand Oaks, California: Sage Publications, Inc. 
Coloma, S., (2012). La migración calificada en América Latina: similitudes y contrastes (Dossier central), Ecuador: FLACSO Sede Ecuador.

DANE, (2012). Boletín de Prensa. Pobreza Monetaria y multidimensional en Colombia, Bogotá: s.n.

DANE, (2014). Estimacion Migración 1985-2020. [En línea] Available at: http://www.dane.gov.co/files/ investigaciones/poblacion/series p85_20/Estimacionesmigracion [Último acceso: 0305 2014].

Diaz, F. O., Ortiz., P., Estrada, S. \& Rodríguez, F., (2012). Los Mashups: aplicaciones compuestas de la Web 2.0, exposición de caso. Ciencias de la Información, pp. $43-48$.

Didou, S., (2008). Presentación. Movilidades académicas y profesionales en América Latina: entre la ignorancia y la polémica., Revista de la educación superior, 37(148), pp. 71-85.

Dixon, M. y otros, (2014). The role of coordinated marketingoperations strategy in services: implications for managerial decisions and execution. Journal of Service Management, pp. 25(2) ,275-294.

Eichentopf, T. K. M. \&. v. S. J., (2011). Modelling customer process activities in interactive value creation. Journal of Service Management, pp. 22(5), 650-663.
Gao, L., Scott, N., Ding, P. \& Coope, C. C., (2012). Tourist Experience Development: Designed Attributes, Perceived Experiences and Customer Value. Strategic Marketing in Tourism Services, pp. 215-230.

Garg, R., Rahman, Z. \& Kumar, I., (2010). Evaluating a model for analyzing methods used for measuring customer experience. Journal of Database Marketing \& Customer Strategy Management, pp. 17(2),78-90.

Grônroos, C., (2007). Service management and marketing (Vol. 2).. New York: Wiley.

Guarnizo, L. E., (2006). El Estado y la migración global colombiana. Migración y desarrollo, pp. (6), 79-101.

Hartono, M., Chuan, T. \& Peacock, J., (2012). Cultural differences in applying Kansei Engineering to services. 2012 Southeast Asian Network of Ergonomics Societies Conference: Ergonomics Innovations Leveraging User Experience and Sustainability, SEANES 2012, p. 0.

Hoyos, G. E., (1981). La migración de profesionales colombianos al exterior. Revista Colombiana de educación, pp. 7-10.

Hsiao K, L., (2013). Android smartphone adoption and intention to pay for mobile internet: Perspectives from software, hardware, design, and value. Library $\mathrm{Hi}$ 
Tech, “31", “2”, “216”, “235”, pp. 2, 31, 216, 235.

Huberman, A. M. \& Miles, M. B., (1994). An expanded sourcebook qualitative data analysis.

Jamali, R., Moshabaki, A., Aramoon, H. \& Alimohammadi, A., (2013). Customer relationship management in electronic environment. Electronic Library, pp. 1, 31, 119, 130.

Kalakota, R. \& Robinson, M., (2003). Services blueprint: roadmap for execution.. s.l.:Addison-Wesley Professional.

Kornblit, A. L., (2007). Metodología cualitativa: Modelos y procedimientos de análisis. Buenos Aires: Biblos.

Kotri, A., (2011). Customer experience evoking and management in services. Tesis Doctoral.

Lee C., -H., Wang Y., -H., Trappey, A. \& Yang S., -H., (2014). Applying geo-social networking and the theory of inventive problemsolving in service innovation and evaluation. Journal of Industrial and Production Engineering, pp. $2,31,95,107$.

Lefebvre, R., (2012). Transformative social marketing: Co-creating the social marketing discipline and brand. Journal of Social Marketing, pp. 2, 118, 129.

Lu., T. \& Bao, J., (2012). A systematic approach to context aware service design. Journal of Computers, pp. 1, 7, 207, 217.
Mager, B., Bender, B. \& Drechsel, P., (2014). A guideline for business mash-ups: Cross-disciplinary partnering as subject for crossdisciplinary research. Touchpoint, pp. 3, 5, 76, 79 .

Maklan, S., (2012). EXQ: a multipleitem scale for assessing service experience. Journal of Service Management, pp. 23(1), 5-33.

Manosso, F. C., Gonçalves, J. M., Alves de Souza, T. \& Bógea, V., (2013). El empleado como gestor de las emociones de los huéspedes. Estudios y perspectivas en turismo, pp. 1-28.

Mateu, E. \& Casal, J., (2003). Tamaño de la muestra. Epidem. Med. Prev, pp. 8-14.

Ministerio de Educación NacionalRepública de Colombia, (2012). Educación Superior 20, Bogotá: Innovasion M\&M LTDA.

Observatorio Laboral para la Educación, (2014). Sistema de Información del Observatorio Laboral. [En línea] Available at: www.graduadoscolombia.edu.co /html/1732/w3-article-195065.h tml [Último acceso: 03-05-2014]

Ochoa, W. M., (2012). Colombia y las migraciones internacionales. Evolución reciente y panorama actual a partir de las cifras. Revista Interdisciplinar da Mobilidade Humana, Issue 39, p. 20(39). 
Pasman, G., (2011). Lost in transition: Issues in designing crossmedia applications and services. SIGDOC'11 - Proceedings of the 29th ACM International Conference on Design of Communication, pp. 175, 179.

PNUD, (2013). Indicadoresinternacionales sobre el desarrollo humano. [En línea] Available at: http://hdr.undp.org/es/datos/ construya/

Ramirez, C. \& Mendoza, L., (2013). Perfil Migratorio de Colombia 2012. Bogotá: Organizacion Internacional para las Migraciones.

Reinoso Fernández-Caparrós, M., (2011). Desarrollo de competencias emocionales en los directivos de una organización a través del outdoor training. Revista Encontros Científicos-Tourism \& Management Studies, pp. 190-197.

Retis, J., (2013). La condición transnacional de las prácticas comunicativas y los retos de la ciudadanía cultural: Latinoamericanos en contextos diaspóricos. Ponencia presentada en el congreso Academia Confronts Emergent Communication Practices and Technopolitics, London: City University.

Riascos, S. C. \& Gonzalez, C. H., (2008). El Poder de la Marca y la Confianza en el E-Business. CollECTeR Iberoamérica 2008, pp. $1-9$.

Rossiter, J. R., (2012). How to Use Qualitative Research to Design a Managerially Useful E-Service Questionnaire. Journal of theoretical and applied electronic commerce research., pp. 52-58.

Schmitt, B., (2007). Experiential marketing. Journal of marketing management, 15(1-3), 53-67. s.l.: Deusto, S.A. Ediciones.

Sirapracha, J. \& Tocquer, G., (2012). Branding and Customer Experience in the Wireless Telecommunication Industry. International Journal of Trade, Economics and Finance, pp. 103-108.

Verhoef, P. C. y otros, (2009). Customer experience creation: determinants, dynamics and management strategies. Journal of Retailing, pp. 31-41.

Walravens, N., (2012). Mobile business and the smart city: developing a business model framework to include public design parameters for mobile city services. Journal of theoretical and applied electronic commerce research, pp. 7(3), 121-135.

Wang, D., Song, Y. \& Zhu, Y., (2010). Information Platform of Smart Grid Based on Cloud Computing. North China Electric Power University, Baoding 071003, p. 22.

Wu L., -W., (2011). Beyond satisfaction: The relative importance of locational convenience, interpersonal relationships, and commitment across service types. Managing Service Quality, pp. 3, 21, 240, 263. 
Yan G, G. \& Qiong, W., (2011). Experience design on $\mathrm{m}$-commerce in china Digital marketing oriented social innovation design. Lecture Notes in Computer Science (including subseries Lecture Notes in Artificial Intelligence and Lecture Notes in Bioinformatics), pp. $427,436$.

Yeh H., J., Chuang L., C. \& Kuo D.C., L., (2013). Applying customer jour- ney methods in SST-based service design: A proposed methodology. Journal of Next Generation Information Technology, pp. 3, 4, 130, 138.

Yu, H., Tang Ko, H. \& Lu, H.-P., (2012). Using Kano's model in designing technology enabled innovative service: An experiential marketing perspective. Orlando, FL., ICEME, p. 3. 
YADY M. GUERRERO FARFÁN \& SANDRA PATRICIA ROJAS BERRÍO 